\title{
LAYOUT OPTIMISATION METHOD FOR ACTIVE STRUCTURAL HEALTH MONITORING
}

\author{
Miroslav Červenka ${ }^{1}$, Rostislav Koštial ${ }^{2}$ \\ ${ }^{1}$ Institute of Aerospace Engineering \\ Brno University of Technology \\ Technická 2, 61669 Brno, Czech Republic \\ cervenka@fme.vutbr.cz \\ ${ }^{2}$ Institute of Aerospace Engineering \\ Brno University of Technology \\ Technická 2, 61669 Brno, Czech Republic \\ kostial@fme.vutbr.cz
}

Keywords: Structural Health Monitoring, Optimal Sensor Placement, Evolutionary Algorithms, Differential Evolution

\begin{abstract}
This paper describes optimisation method for designing sensoric layout for Active Structural Health Monitoring (A-SHM) by Ultrasonic Guided Waves (UGW) on metal and non-metal (composite) materials. The SHM sensors need to be placed optimally in order to detect structural damage with high probability before the damage turns critical. Configuration of used optimisation algorithm for such task is not straightforward. Differential Evolution (DE) has two configuration parameters - the mutation factor $\mathrm{F}$ and the crossover rate $\mathrm{CR}$ - whose settings largely influence the solution quality the optimisation process can yield. For that matter we describe an elaborated a method to guide this selection towards good results using visual heat maps with the intent to select best DE's variant and particular configuration to receive the most optimal SHM sensorics layout.
\end{abstract}

\section{INTRODUCTION}

Structural Health Monitoring (SHM) is the process of implementing a damage detection strategy on structures. This paper is focused on the Structural Health Monitoring (SHM) by the Ultrasonic Guided Waves (UGW) which are researched at the Brno University of Technology on the Institute of Aerospace Engineering.

Typical materials used in structural design are natural waveguides. Therefore, SHM using Ultrasonic Guided Waves such as Lamb waves for active sensing provide great opportunity to obtain information about the current state of structures. The most challenging issue is the optimal placement of sensor/ actuator on structure with limited number of actuators/sensors while covering the entire surface of the monitored structure.

There are many studies on optimal sensor placement, but only few of them are viable for UGW or Lamb-wave SHM. This paper provides a performance comparison of two optimization methods which are viable for UGW or Lamb-wave SHM. Methods independent for metal and non-metal (composite) materials are considered. 


\section{SHM IN AIRCRAFT MAINTENANCE PROGRAMS}

Aircraft maintenance is linked to aviation programs since the days of the first attempts of man controlled flight. Aircraft maintenance and approach to maintenance has undergone significant evolution since the beginning of aviation. From repairs after failure through simple maintenance plans to modern approaches based on reliability of aircraft parts and systems such as Reliability Centered Maintenance or Maintenance Steering Group (MSG-3). With expanding fleets aircraft maintenance becomes an immense issue with the ever increasing demands on the effectiveness, cost-efficiency and reliability. In the domain of aircraft structures maintenance many various methods of SHM are being considered and also practically tested with the aim to completely replace the error-prone human element in aircraft structure inspection tasks in the foreseeable future [1].

Our approach to the SHM problem can be spotted in employment of ultrasonic guided waves (UGW). These waves are excited by piezoelectric actuators/sensors and can provide a highly efficient method for the Non-Destructive Evaluation (NDE) and SHM of generic aircraft structures. Especially in the case of using for Automated Structural Health Monitoring (A-SHM) at part of structure where access is limited of even impossible to common inspection methods. Guided acoustic waves in the range between $100-300 \mathrm{kHz}$ have unique detection capability which combines reasonable sensitivity to damage with reasonably good propagation range. Guided waves can propagate over several meters along the examined structure set under appropriate conditions, yielding rather good damage sensitivity [2]. There are many books and papers with focus to this issue which can give detailed information on Ultrasonic Guided and Lamb Waves [3, 4].

There are many examples established in the area of Non-Destructive Testing (NDT) industry using Guided Wave Systems for inspection of pipelines and rails $[5,6]$. In the aviation industry there are investigated techniques for use of Guided Waves for A-SHM on metal and non-metal materials, especially on composites $[7,8]$. Typically, an array of sensors is permanently attached to the structure and used to emitting UGW energy. The same array can then be used to catch the signals from other sensors.

The key here is to attach limited number of the SHM sensors on well-chosen hot-spots on the aircraft structure with the additional aim to restrain weight and complexity of the entire system while keeping the SHM levels on the highest coverage levels possible on the area in consideration. This SHM task has thus become an optimal sensor placement problem which can be treated with existing set of evolutionary algorithms.

\section{EVOLUTIONARY ALGORITHMS}

Evolutionary algorithms (EA) based on principles of natural selection belong to very efficient methods of global optimization. They are successfully used in many engineering applications. In general, EAs are able to find a feasible solution of an optimization problem in a reasonable time. However, when using them for complex problems, the time required for finding a suitable solution might be unacceptably long. Therefore, many studies were published and lots of experiments were undertaken in effort to speed-up evolutionary algorithms. During the last decade, parallelisation became one of the ways of improving the computational performance of evolutionary algorithms [9].

Furthermore, efficiency of evolutionary algorithms is strongly dependent on correct setting of their control parameters. Standard attempt in applications is to set up the values of control parameters by tuning via trial-and-error preliminary experiments. This is time-consuming and does not satisfy the user's natural requirement for quick and reliable heuristic search algorithm, which should be efficient enough to find global optimum without requiring any deeper user's knowledge. [10] 


\subsection{Genetic Algorithm}

As described in [11], in a genetic algorithm, a population of candidate solutions (called individuals, creatures, or phenotypes) to an optimization problem is evolved toward better solutions. Each candidate solution has a set of properties (its chromosomes or genotypes) which can be mutated and altered; traditionally, solutions are represented in binary as strings of $0 \mathrm{~s}$ and $1 \mathrm{~s}$, but other encodings are also possible. [12]

The evolution usually starts from a population of randomly generated individuals, and is an iterative process, with the population in each iteration called a generation. In each generation, the fitness of every individual in the population is evaluated; the fitness is usually the value of the objective function in the optimization problem being solved. The more fit individuals are stochastically selected from the current population, and each individual's genome is modified (recombined and possibly randomly mutated) to form a new generation. The new generation of candidate solutions is then used in the next iteration of the algorithm. Commonly, the algorithm terminates when either a maximum number of generations has been produced, or a satisfactory fitness level has been reached for the population.

For our experiments the default Matlab implementation of GA has been used.

\subsection{Differential Evolution}

The Differential Evolution (DE) as proposed by Storn and Price [13] is a powerful optimisation technique designed for global optimisation. It can be used as a general optimiser, yet its qualities emerge in assignments where conventional optimisation techniques fail. There are many successful engineering applications of this indeed very strong algorithm like multiprocessor synthesis, neural network learning, radio network design or plasma reactor optimisation.

DE does not explicitly rely on gradient of the optimised problem, it works with multiple agents collaborating in a direct-search manner, treating the optimisation problem as a black-box, which merely emits a measure of fitness for candidate solutions. DE then creates new candidate solutions by combining solutions according a variant-specific chain of rules (see Table 1) from its current set of agents (population), accepting new solutions in case of fitness improvement.

DE treats its population in iteration loops called generations. In each generation every agent (a candidate solution - vector of parameters for the optimised model; an individual) is manipulated in order to increase its fitness. For example, in the variant $D E /$ best/ 1 two randomly chosen individuals from the population are subtracted one from another. This product undergoes a mutation (multiplied by the mutation factor $F$ ) giving us a weighted differential vector. Consequently, this is added to the best individual from the population (having the best fitness) resulting into a noise vector (denoted as $v$ in the Table 1). From this point, the procedure is then the same for all variants. According the crossover rate $(C R)$, items from the current (modified) individual and the noise vector are selected in order to produce a trial vector (or trial individual), which is evaluated by the cost function. In case its fitness is better than fitness of the source individual, it survives into the next generation, while the weaker individual does not.

\begin{tabular}{lcc}
\hline & DE Variant & Formula \\
\hline 1 & DE/best/1 & $v=x_{\text {best }, j}^{G}+F \cdot\left(x_{r_{1}, j}^{G}-x_{r_{2}, j}^{G}\right)$ \\
2 & DE/rand/1 & $v=x_{r_{1}, j}^{G}+F \cdot\left(x_{r_{2}, j}^{G}-x_{r_{3}, j}^{G}\right)$ \\
3 & DE/rand-to-best/1 & $v=x_{i, j}^{G}+\lambda \cdot\left(x_{\text {best,j }}^{G}-x_{i, j}^{G}\right)+F \cdot\left(x_{r_{1}, j}^{G}-x_{r_{2}, j}^{G}\right)$ \\
4 & DE/best/2 & $v=x_{\text {best,j }}^{G}+F \cdot\left(x_{r_{1}, j}^{G}+x_{r_{2}, j}^{G}-x_{r_{3}, j}^{G}-x_{r_{4}, j}^{G}\right)$ \\
5 & DE/rand/2 & $v=x_{r_{5}, j}^{G}+F \cdot\left(x_{r_{1}, j}^{G}+x_{r_{2}, j}^{G}-x_{r_{3}, j}^{G}-x_{r_{4}, j}^{G}\right)$ \\
\hline
\end{tabular}

Table 1: Differential Evolution variants 
Differential Evolution is considered to be one of the most powerful evolutionary algorithms of present days. Besides its good convergence properties, the main advantage of DE lies with its conceptual simplicity, ease of use and low number of control parameters. However, like any other evolutionary algorithm, the success of DE is also very sensitive on setting of its control parameters. There are three DE's control parameters: (1) the population size NP, (2) the mutation factor $F$ (a real-value factor that controls amplification of differential variations) and (3) the crossover factor $C R$ (also a real value, controlling the crossover operation).

As the correct configuration of $(F, C R)$ has crucial influence on DE's performance, many literature entries offer starting, default or recommended values. In [10] many of these are summarised, however, as later experiments had proven there is no rigid rule one can follow. Every particular optimised problem needs to have $(F, C R)$ mapped to find the ones giving best performance and results every or at least most of the time.

\section{THE OPTIMISATION TASK}

It has been discovered [14] that in anisotropic materials the pattern of sound wave dissemination has an approximate shape of an ellipse (or a circle in isotropic 2D environment, see Figure 1). For that matter this same shape has been used in the experiments described later on.

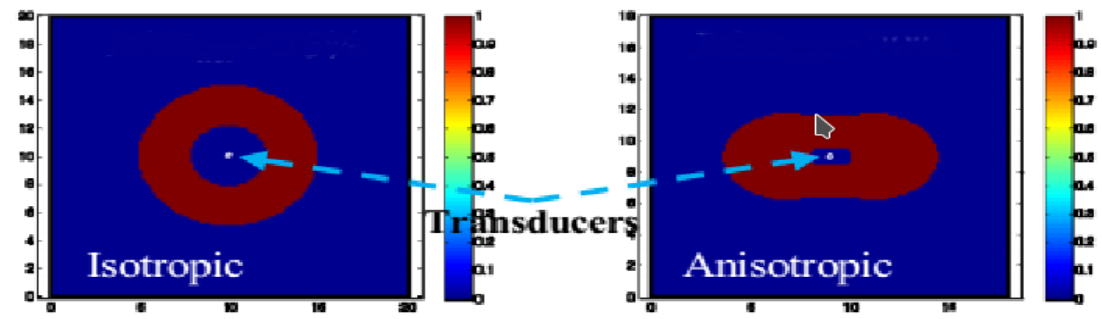

Figure 1: Signal dissemination pattern in isotropic and anisotropic materials [14]

Uniform section of $500 \times 500$ units ( $\mathrm{mm}, \mathrm{cm}$, pixels/px in this case) is to be populated by SHM sensors in such way the entire (or maximal) area is within range of the sensors. The sensor range (dimension of the ellipses) was defined as [width $\mathrm{x}$ height] $=[60 \times 120] \mathrm{px}$ while the shapes could be rotated by $45^{\circ}$ in steps $<0 ; 45 ; 90 ; 135 ; 180 ; 225 ; 270 ; 315>^{\circ}$. Number of sensors was configured as 40 . Physical dimensions range from 6 to $20 \mathrm{~mm}$ in diameter. For these simulations, however, these values are not substantial.

Please note, that this is a very simplified mapping of sensors influencing just one other sensor pairs (transducer sensors). A more complex scenario where all sensors will have impact on all adjoining ones will follow.

\subsection{Optimisation Results}

Both evolutionary algorithms were executed multiple times and the best achieved results are presented in Figure 2. The Genetic Algorithm driven by Matlab's implementation was heavily supported by injection of fresh individuals into the population in the moment the algorithm started to stagnate (i.e. population's diversity was low). This feature contributes significantly to find reasonably good solution to the optimised problem. 


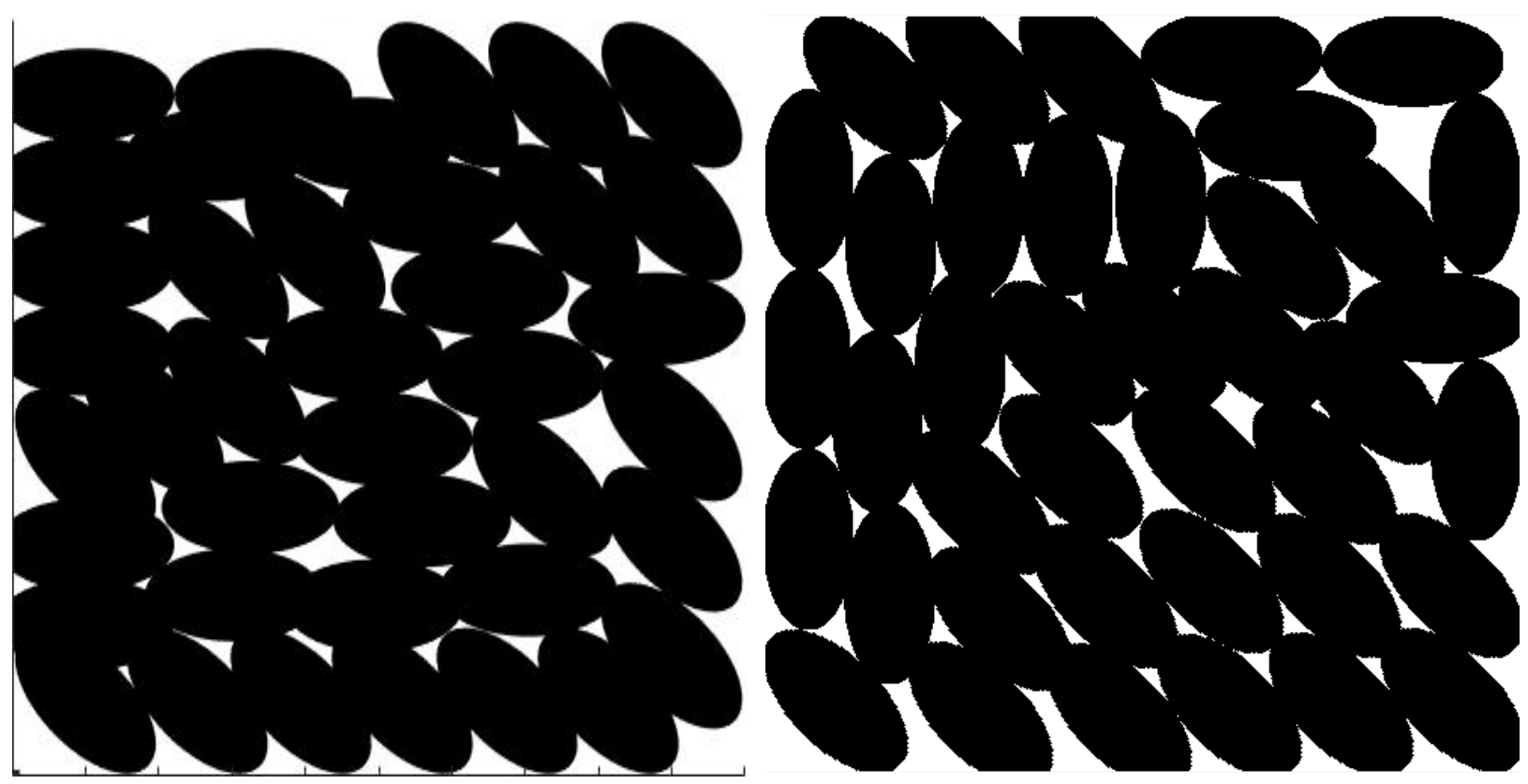

Figure 2: Sensor placement results - LEFT: Genetic Algorithm (87.4\% of coverage), RIGHT: Differential Evolution (88.2\% of coverage)

As Differential Evolution in used implementation does not possess any such feature (there is nothing like that defined in the DE realm), its configuration parameters play crucial role in the final result. Such dependency can be clearly indicated in a simple heatmap (see Figure 3). The best solutions for this task have been raised for $\mathrm{DE}$ in configuration with $\mathrm{F}=0.1, \mathrm{CR}=0.4$.

Why is creating heatmaps before running full optimisation so useful? As described in [10] the DE configuration parameters are application specific up to such extent where generally recommended values (which also vary author by author) raise only average results or even completely preposterous ones. Figure 4 depicts some examples of how strongly can DE convergency depend on its configuration parameters when executed for dissimilar optimisation tasks. There you can clearly see how much vary the areas of best results between different variants of $D E$ and given cost functions. Darker colours represent better solutions yielded for particular pair of $(F, C R)$. White crosshairs indicate best set of ( $F$, CR) for particular experiment configuration. 


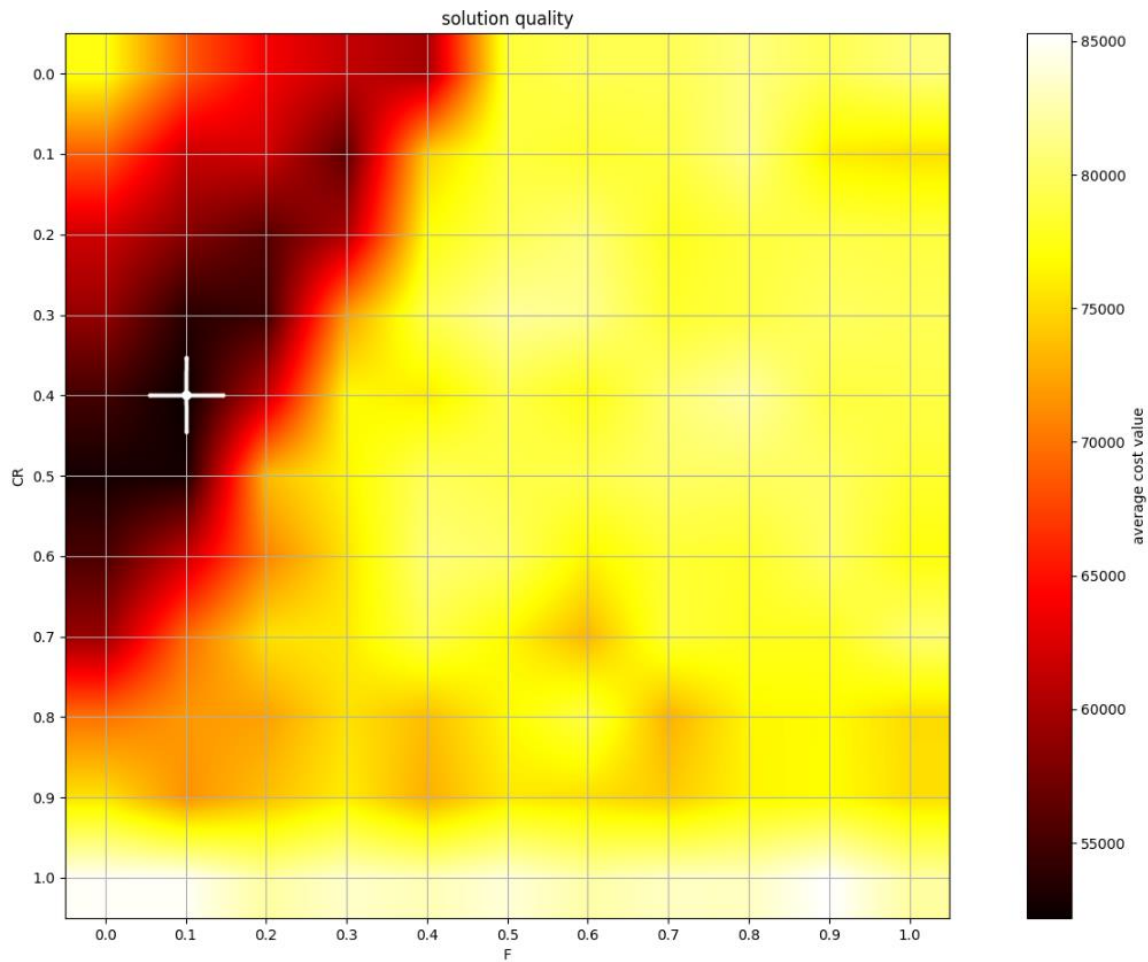

Figure 3: DE configuration influence on the optimisation result yielded (darker is better; best configuration marked by white crosshair)
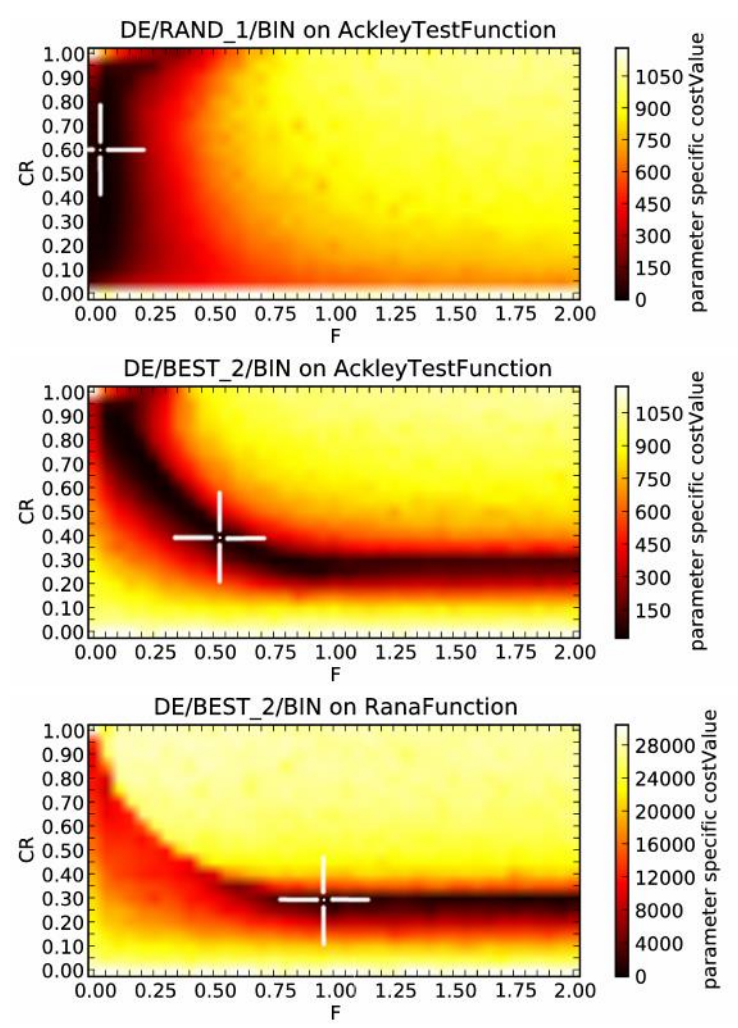
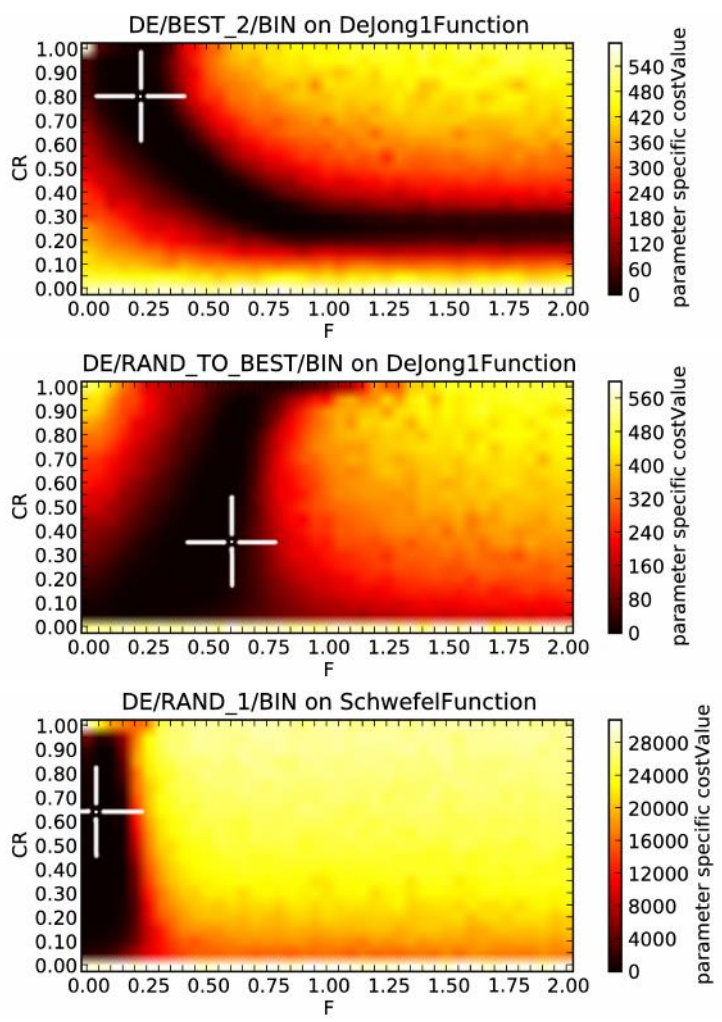

Figure 4: DE solution quality as function of $F$ an $C R$ control parameters (darker is better; best configuration marked by white crosshair) 


\section{CONCLUSIONS}

In this contribution two optimisation methods for placement of sensors for structural health monitoring based on ultrasonic guided waves propagation were presented. For this task it is necessary to reflect specific application needs, mainly the material structure of composite sections. Evolutionary algorithms have proven to be very successful in task of sensor placement. In case of Differential Evolution it has been demonstrated that setting of the mutation factor $\mathrm{F}$ and crossover rate $\mathrm{CR}$ to their perfectly correct values is essential to receive a good optimisation result while generally recommended configuration values would result into massive optimisation failure.

\section{ACKNOWLEDGEMENTS}

These outcomes were supported by the project TE02000032 - Advanced Aerostructures Research Centre. This project was realized with financial support from national budget of the Technology Agency of the Czech Republic.

\section{REFERENCES}

[1] Koštial, R.; Janhuba, L.; Hlinka, J. (2017.) Intelligent Scheduled Maintenance Methodology For General Aviation Structures Based On Msg-3 And Multiple-Criteria Decision Making Analysis. In Engineering Mechanics 2017. p. 498-501. ISBN: 978-80-214-5497-2. ISSN: 1805-8248.

[2] Taha M., M. Reda, Aboelmaged Noureldin, J. L. Lucero and Thomas J. Baca. (2006). Wavelet Transform for Structural Health Monitoring: A Compendium of Uses and Features. In Structural Health Monitoring 2006, p. 267-295, Sage Publications

[3] Joseph L. Rose. (2014) Ultrasonic Guided Waves in Solid Media, Chapter 1.4: The difference between Structural Health Monitoring (SHM) and Nondestructive Testing (NDT), Cambridge University Press. ISBN: 978-1107048959

[4] Giurgiutiu V. (2014). Structural health monitoring with piezoelectric wafer active sensors. Amsterdam: Academic Press, an imprint of Elsevier. ISBN: 978-0-12-418691-0

[5] Peter Cawley, P. D. Wilcox, D. N. Alleyne, Brian Pavlakovic, Matthew Evans, Kathryn Vine and Michael J. S. Lowe. (2004). Long Range Inspection of Rail Using Guided Waves-field Experience. In Proceedings of the 16th World Conference on Non-Destructive Testing, Montreal, Canada.

[6] Moon Ho Park, In Sup Kim, Young Ku Yoon. (1996). Ultrasonic inspection of long steel pipes using Lamb waves, NDT \& E International, Volume 29, Issue 1, Pages 13-20, ISSN 0963-8695

[7] Hyun Woo Park, Hoon Sohn, Kincho H. Law, Charles R. Farrar. (2007). Time reversal active sensing for health monitoring of a composite plate, Journal of Sound and Vibration, Volume 302, Issues 1-2, Pages 50-66, ISSN 0022-460X

[8] George Zhao, Huidong Gao, Guangfan Zhang, Bulent Ayhan, Fei Yan, Chiman Kwan, Joseph L. Rose. (2007). Active health monitoring of an aircraft wing with embedded piezoelectric sensor/actuator network: I. Defect detection, localization and growth monitoring. Smart Materials and Structures. 16. 1208.

[9] Červenka, M. (2006). Distributed Evolutionary Algorithms. Ph.D. thesis.

[10] Červenka, M., Boudná, H. (2018). Visual Guide on F and CR Parameteres Influence on Differential Evolution Solution Quality. In 24th International Conference ENGINEERING MECHANICS 2018

[11] Genetic Algorithm Wiki [online] [cit. 13.8.2018]

[12] Whitley, D. (1994). A genetic algorithm tutorial. In: Statistics and Computing, p. 65-85. ISSN 15731375

[13] Storn, R., Price, K. (1997). Differential Evolution - A Simple and Efficient Heuristic for global Optimization over Continuous Spaces. In: Journal of Global Optimization, p. 341-359, December 1997 - Vol. 11 
[14] V. Janapati, K. Lonkar, F.-K. Chang. (2012). Design of Optimal Layout of Active Sensing Diagnostic Network for Achieving Highest Damage Detection Capability in Structures, 6th European Workshop on Structural Health Monitoring.

[15] MSG-3, https://www.skybrary.aero/index.php/Maintenance_Steering_Group-3_(MSG-3), [online] [cit. 16.10.2018]

\section{COPYRIGHT STATEMENT}

The authors confirm that they, and/or their company or organization, hold copyright on all of the original material included in this paper. The authors also confirm that they have obtained permission, from the copyright holder of any third party material included in this paper, to publish it as part of their paper. The authors confirm that they give permission, or have obtained permission from the copyright holder of this paper, for the publication and distribution of this paper as part of the READ 2018 proceedings.

This is an open access article distributed under the Creative Commons Attribution License which permits unrestricted use, distribution, and reproduction in any medium, provided the original work is properly cited. (CC BY 4.0). 